\title{
Antimicrobial Resistance in Carbapenem Resistance Pseudomonas
}

\author{
SONIA TAHIR ${ }^{1}$, SAADIA CHAUDHARY², TAHIR NAEEM ${ }^{3}$. \\ 1,2 Department of Pathology, Lahore Medical \& Dental College, Lahore \\ ${ }^{3}$ Department of Pathology, University of Lahore \\ Correspondence to Dr. Sonia TahirEmail:Sonia.tahir@lmdc.edu.pk, Cell:0321 8133444.
}

\begin{abstract}
Aim: To figure out the antimicrobial sensitivity effect of multidrug resistant Pseudomonas aeruginosa obtained from several type of clinical specimens.

Study setting: Department of Microbiology and Resource laboratory, University of Health Sciences Lahore. Methods: A sum total of 53 isolates of multi-resistant Pseudomonas aeruginosa were selected from Jinnah hospital Lahore during the period of $1^{\text {st }}$ January 2016 to $2^{\text {nd }}$ February 2017.

Nutrient agar slants were used for the transportation of resistant strains. In accordance with the CLSI manuals re-confirmation and processing of the strains were accomplished. The sub culturing and incubation was done on culture media such as MacConkey and blood agar at room temperature for 1 day. Standard confirmation of isolates under went by the graded morphological, cultural and biochemical techniques. In order to achieve this, Gram staining, culture media such as blood, oxidase test, motility test were executed.

Results: The resistance pattern of Pseudomonas aeruginosa against antibiotics was as follows: Meropenem $53(100 \%), 51(96 \%)$ to piperacillin-tazobactam, $49(92 \%)$ to ceftazidime, $43(81 \%)$ to amikacin, $41(77 \%)$ showed resistance to aztreonam, $48(91 \%)$ to quinolones as shown in figure. Almost all the Pseudomonas aeruginosa were resistant to aztreonam except for $23 \%$ ( $n=12$ isolates). Colistin was predominant as the major strength of treatment for Pseudomonas aeruginosa with sensitivity of $48(91 \%)$.

Keywords: Disk-diffusion, Carbapenem, McFarland.
\end{abstract}

\section{INTRODUCTION}

Antimicrobial resistance emergence and spread continues to threaten our ability to treat common infections ${ }^{1}$. According to $\mathrm{WHO}$, antimicrobials is one of the prominent global health threat imposed on humanity ${ }^{2}$. Recent issue of growing concern is the deadly spread of carbapenem resistant organisms also known as "superbugs"3. The cost of carbapenem resistant microbes to national economies and health system alters treatment of patients, lead to extensive hospital stays and need for more expensive, intensive treatment ${ }^{4}$.

The objective of the study was to figure out the antimicrobial sensitivity effect of multidrug resistant Pseudomonas aeruginosa obtained from several type of clinical specimens.

\section{METHODOLOGY}

Drug susceptibility By Disk-diffusion: First of all bacterial growth was encouraged in TSB at $35^{\circ} \mathrm{C}$ for 1 day. After that, bacteria were added to emulsion sample in comparisons to $0.5 \mathrm{McF}$ arland standards. With sterilized cotton swabs the antibiotic disks cropped up over the $\mathrm{MH}$ media for 1 day at $35^{\circ} \mathrm{C}$. Following growth period, length of the zone of inhibition were assessed. By Kirby-Bauer testing phenotypic characterization of isolates were determined. Susceptibility testing was executed as stated by standardized CLSI 2015 protocols in all the multidrug resistant isolates ${ }^{5}$. Pseudomonas aeruginosa ATCC27853 was used as control for the susceptibility tests. Chosen antibacterial for Pseudomonas aeruginosa amikacin (AK), ceftazidime (CAZ), ciprofloxacin (CIP), Meropenem (MEM), aztreonam (ATM) and piperacillin/tazobactam (TZP) ${ }^{3,6}$. The zone of complete inhibition of colistin for Pseudomonas species had also been performed by Kirby diffusion technique.

\section{RESULTS}

Antimicrobial susceptibility of clinical isolates. The isolates used in this study were ineffective to a minimum of three group of antibacterial like penicillins, cephalosporin, aminoglycosides, quinolones and carbapenems. All the isolates $(n=53)$ were found to be resistant to carbapenem thus extensively drug resistant (XDR). Isolates of Pseudomonas were irresponsive to amikacin, ciprofloxacin, ceftazidime, piperacillin-tazobactam and Meropenem. The resistance pattern of Pseudomonas aeruginosa against antibiotics was as follows: Meropenem 100\% (53 isolates), 96\% (51 isolates) to piperacillin-tazobactam, 92\% (49 isolates) to ceftazidime, $81 \%$ (43 isolates) to amikacin, $77 \%$ (41 isolates) exhibited defiance to aztreonam, 91\% (48 isolates) to quinolones as depicted by the figure. A majority of Pseudomonas aeruginosa were irresponsive to aztreonam, excluding $23 \% 12$ isolates a clear indication of presence of additional carbapenemase as MBL are particularly ineffective to aztreonam ${ }^{7}$. Colistin was predominant as the major strength of treatment for Pseudomonas aeruginosa with sensitivity of $48(91 \%)$. Except for the $5(9 \%)$ isolates expressing pan drug resistance, colistin succeeded as drug of choice amongst the Pseudomonas considered in our study as can be seen in chart I.

Received on 28-03-2021

Accepted on 11-08-2021 
Figure I: Level of sensitivity pattern of Pseudomonas aeruginosa to various antibiotics.

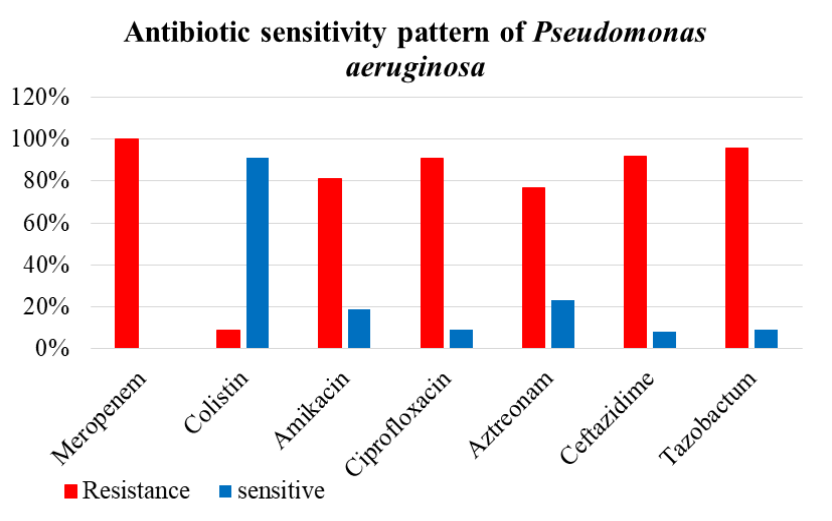

\section{DISCUSSION}

High proportion of Pseudomonas were resistant to piperacillin/tazobactam, levofloxacin, aztreonam, ceftazidime, ciprofloxacin. One possibility against emergence of resistance towards antibiotics is if people do not change the way antibiotic are used therefore will lead to upcoming intrinsic resistance in Pseudomonas aeruginosa against the drugs ${ }^{8}$. Reportedly $19 \%$ amikacin sensitivity levels displayed on the other hand, this decreasing trend of amikacin is lowered than the conclusion drawn by Cavallo et al in 2007 and Hussain et al in 2015 who remarked 86\% and $56 \%$ aminoglycoside susceptibility trends ${ }^{9,10}$. Currently overuse of aminoglycosides against gram negative bacteria in our hospital setups and dissemination of these pathogens by inappropriate hygienic measures is probably the reason ${ }^{10}$. We also concluded that aztreonam drug level in Pseudomonas aeruginosa was $88 \%$ in regulation with conclusive studies of Peymani et al in 2011 (95\%), kalam et al in 2012 (92\%), and Ameen et al in $2011(86 \%)$ 11,12,13. Resistance to all $\beta$-lactam drugs was imparted by MBL while excluding monobactams, such as aztreonam. However the aztreonam resistance observed in our study suggest some other modes of resistance such as concurrent presence of varied $\beta$-lactamases particularly the lactamases. Colistin remains as the mainstay of treatment for Pseudomonas with result shown up to of $91.8 \%$. Seen in the studies of Jeya $\mathrm{M}$ et al in 2014 similar trends of $95 \%$ susceptibility towards Pseudomonas had been noted ${ }^{14}$. Whereas $5(9 \%)$ of Pseudomonas aeruginosa exhibiting phenomenon of pan-drug resistance. These pan drug resistant strains are apparent because colistin will suffer the same fate as the previous antibiotics and become ineffective ${ }^{16}$

Moreover, the horizontal transfer of resistance gene through plasmids amongst microbes are the key against the acquirement of resistance to various drug under cosideration $^{20}$. Carbapenem the best medication for multidrug resistant Gram-negative pathogens lead to it's over used in hospital ICUs contributing to increase carbapenem resistance ${ }^{17}$.

Meropenem 53(100\%), colistin 5(9\%), amikacin 43(81\%), ciprofloxacin 48(91\%), aztreonam 41(77\%), ceftazidime $49(92 \%)$, tazobactam 51(96\%).

\section{CONCLUSION}

To conclude, Colistin is the only last resort treatment for life-threatening infections caused by carbapenem resistant Pseudomonas. This was also analyzed that bacteria resistant to colistin for which no effective antibacterial present currently.

\section{REFERENCES}

1. Royer, S., Faria, A. L. S., Seki, L. M., Chagas, T. P. G., De Campos, P. A., Da Fonseca Batistão, D. W., Asensi, M. D., Gontijo Filho, P. P. \& Ribas, R. M. 2015. Spread of multidrug-resistant Acinetobacter baumanii and Pseudomonas aeruginosa clones in patients with ventilator-associated pneumonia in an adult intensive care unit at a university hospital. Braz. J. Infect. Dis. 19: 350-357.

2. World Health Organization. 2013. Media Centre, Antimicrobial Resistance, Fact Sheet No194 [Online]. Available: Http:/Www.Who.Int/Mediacentre/Factsheets/Fs194/En/ [Accessed 22 September 2015

3. Akya, A., Salimi, A., Nomanpour, B., Ahmadi, K. 2015. Prevalence and clonal dissemination of Metallo- $\beta$-Lactamase-producing Pseudomonas aeruginosa in Kermanshah. Jundishapur. J Microbiol. 8(7).

4. Balkhy, H. H., El-Saed, A., Maghraby, R., Al-Dorzi, H. M., Khan, R., Rishu, A. H. \& Arabi, Y. M. 2014. Drug-resistant ventilator associated pneumonia in a Tertiary Care Hospital in Saudi Arabia. Ann. Thorac. Med. 9(2): 104-111.

5. Clinical and Laboratory Standards Insitute (CLSI). 2015. Performance standards for antimicrobial susceptibility testing. page no.112-118.

6. Anwar, M., Ejaz, H., Zafar, A. \& Hamid, H. 2015. Phenotypic detection of Metallo- $\beta$-lactamases in carbapenem resistant Acinetobacter baumanii isolated from pediatric patients in Pakistan. Journal of Pathogens 6pgs.

7. Amudhan, M. S., Sekar, U., Kamalanathan, A. \& Balaraman, S. 2012. Blaımp and blavim mediated carbapenem resistance in Pseudomonas and Acinetobacter species In India. J. Infect. Dev. Ctries. 6(11): 757762.

8. Asghar, A. H. 2012. Antimicrobial susceptibility and metallo- $\beta$ lactamase production among Pseudomonas aeruginosa isolated from Makkah hospitals. Pak.J. Med. Sci. 28(5): 781-786.

9. Cavallo, J., Hocquet, D., Plesiat, P., Fabre, R. \& Roussel-Delvallez, M. 2007. Susceptibility of Pseudomonas aeruginosa to antimicrobials: A 2004 French multicenter Hospital Study. J. Antimicrob. Chemother. 59(5): 1021-1024.

10. Abbas, S.H., Khan, M.Z., Naeem, M., Adil, M., Naz, S.M., Khan, A., Khan, M.U. 2015. Sensitivity patterns of Pseudomonas aeruginosa isolates obtained from clinical specimen in Peshawar. J. Ayub Med. Coll. Abbottabad. 27(2).

11. Peymani, A., Nahaei, M.-R., Farajnia, S., Hasani, A., Mirsalehian, A. Sohrabi, N., Abbasi, L. 2011. High prevalence of Metallo- $\beta$-Lactamaseproducing Acinetobacter baumanii in a Teaching Hospital in Tabriz, Iran. Jpn J. Infect. Dis. 64: 69-71.

12. Kalam, K., Qamar,F., Kumar,S., Ali,S., Baqi,S. 2012. Risk factor for carbapenem resistant bacteraemia and mortality due to gram negative bacteria in a developing country. J. Pak. Med. Assoc. 64(5).

13. Ameen, N., Memon, Z., Shaheen, S., Fatima, G. \& Ahmed, F. 2015 Imipenem resistant Pseudomonas aeruginosa: The fall of the final quarterback. Pak. J. Med. Sci. 31(3): 561-565.

14. Kandasamy, S., Vijayalakshmi, K., Jeya M. 2014. Prevalence of multidrug resistant bacteria and blakPC-2 gene detection of carbapenem resistant isolates from clinical samples of intensive care units in a tertiary care hospital. Int. J. Res. Health Sci. 2(1): 201-6.

15. Diene, S. M. \& Rolain, J.-M. 2014. Carbapenemase genes and genetic platforms in Gram-negative bacilli: Enterobacteriaceae, Pseudomonas and Acinetobacter Species. Clin. Microbiol. Infect. 20(9): 831-838.

16. Da Silva, G. J. \& Domingues, S. 2016. Insights on the horizontal gene transfer of carbapenemase determinants in the opportunistic pathogen Acinetobacter baumanii. Antimicrob. Agents Chemother. 45(2): 546552.

17. Tsakris, A., Ikonomidis, A., Poulou, A., Spanakis, N., Vrizas, D., Diomidous, M., Pournaras, S. \& Markou, F. 2008. Clusters of imipenem-resistant Acinetobacter baumanii clones producing different carbapenemases in an intensive care unit. Clin. Microbiol. Infect. 14: 588-594.. 